Electronic structure, Born effective charges and spontaneous polarization in magnetoelectric gallium ferrite

This article has been downloaded from IOPscience. Please scroll down to see the full text article.

2011 J. Phys.: Condens. Matter 23325902

(http://iopscience.iop.org/0953-8984/23/32/325902)

View the table of contents for this issue, or go to the journal homepage for more

Download details:

IP Address: 14.139.60.97

The article was downloaded on 17/08/2011 at 06:28

Please note that terms and conditions apply. 


\title{
Electronic structure, Born effective charges and spontaneous polarization in magnetoelectric gallium ferrite
}

\author{
Amritendu Roy ${ }^{1}$, Somdutta Mukherjee ${ }^{2}$, Rajeev Gupta ${ }^{2,3}$, \\ Sushil Auluck $^{4}$, Rajendra Prasad ${ }^{2}$ and Ashish Garg ${ }^{1}$ \\ ${ }^{1}$ Department of Materials Science and Engineering, Indian Institute of Technology, \\ Kanpur-208016, India \\ ${ }^{2}$ Department of Physics, Indian Institute of Technology, Kanpur-208016, India \\ ${ }^{3}$ Materials Science Programme, Indian Institute of Technology, Kanpur-208016, India \\ ${ }^{4}$ National Physical Laboratory, Dr K S Krishnan Marg, New Delhi-110012, India \\ E-mail: ashishg@iitk.ac.in
}

Received 19 April 2011, in final form 5 July 2011

Published 25 July 2011

Online at stacks.iop.org/JPhysCM/23/325902

\begin{abstract}
We present a theoretical study of the structure-property correlation in gallium ferrite, based on first-principles calculations followed by a subsequent comparison with experiments. The local spin density approximation (LSDA $+U$ ) of the density functional theory has been used to calculate the ground state structure, electronic band structure, density of states and Born effective charges. The calculations reveal that the ground state structure is orthorhombic $P c 2_{1} n$ having A-type antiferromagnetic spin configuration, with lattice parameters matching well with those obtained experimentally. Plots of the partial density of states of constituent ions exhibit noticeable hybridization of $\mathrm{Fe} 3 \mathrm{~d}, \mathrm{Ga} 4 \mathrm{~s}, \mathrm{Ga} 4 \mathrm{p}$ and $\mathrm{O} 2 \mathrm{p}$ states. However, the calculated charge density and electron localization function show a largely ionic character of the $\mathrm{Ga} / \mathrm{Fe}-\mathrm{O}$ bonds which is also supported by a lack of any significant anomaly in the calculated Born effective charges with respect to the corresponding nominal ionic charges. The calculations show a spontaneous polarization of $\sim 59 \mu \mathrm{C} \mathrm{cm}^{-2}$ along the $b$-axis which is largely due to asymmetrically placed $\mathrm{Ga} 1, \mathrm{Fe} 1, \mathrm{O} 1, \mathrm{O} 2$ and $\mathrm{O} 6$ ions.
\end{abstract}

(Some figures in this article are in colour only in the electronic version)

\section{Introduction}

Gallium ferrite $\left(\mathrm{GaFeO}_{3}\right.$ or $\left.\mathrm{GFO}\right)$ is a piezoelectric and a ferrimagnet with its magnetic transition temperature close to room temperature $(\mathrm{RT})[1,2]$. The transition temperature is affected largely by the Ga:Fe ratio within the single phase region $\left(0.7 \leqslant x \leqslant 1.4\right.$ for $\left.\mathrm{Ga}_{2-x} \mathrm{Fe}_{x} \mathrm{O}_{3}\right)$ and can be tuned to the values above RT [1,3-5]. As a result, accompanied by a good piezoelectric response [6], compositionally modulated GFO is an exciting RT magnetoelectric material. Initial structural studies on this compound predicted the structure to be orthorhombic with $P c{ }_{1} n$ symmetry $[4,7,8]$, confirmed subsequently by recent studies using neutron $[1,2,9,10]$ and $\mathrm{x}$-ray diffraction $[1,3,10,11]$ investigations made on both powder and single crystals over a wide temperature range $(4-700 \mathrm{~K})$. The orthorhombic unit cell comprises eight formula units and the RT lattice parameters are $a=$ $8.7512 \AA, b=9.3993 \AA, c=5.0806 \AA$ [8]. The unit cell contains two nonequivalent $\mathrm{Ga}$ and $\mathrm{Fe}$ sites and six nonequivalent $\mathrm{O}$ sites. While $\mathrm{Ga} 2, \mathrm{Fe} 1$ and $\mathrm{Fe} 2$ ions are octahedrally coordinated by oxygen, Ga1 ion has tetrahedral coordination [1]. However, experimental observations reveal considerable cation site disorder indicating partial occupancy of $\mathrm{Ga}$ and $\mathrm{Fe}$ sites by $\mathrm{Fe}$ and $\mathrm{Ga}$ ions, respectively $[1,8]$. The cation site disorder is also believed to be responsible for observed ferrimagnetism in GFO [1]. Although not much has been reported on the structural distortion in GFO, the asymmetric nature of the $\mathrm{Ga} 1-\mathrm{O}$ tetrahedron is believed to contribute to the piezoelectricity in GFO with its piezoelectric coefficient being almost double that of quartz [12]. 
Despite a series of experimental studies, theoretical work, especially first-principles-based calculations on GFO, has not really progressed, presumably because of the complex crystal structure and partial site occupancies of the cations. The only report by Han et al [13] emphasizes the magnetic structure and spin-orbit coupling behavior using the linear combination of localized pseudoatomic orbitals (LCPAO) method. However, there are no reports on the theoretical understanding of the structure, bonding and Born effective charges of GFO which are crucial to elucidate the structural distortion, nature of bonds and resulting polarization in GFO. Here, we present a first-principles density functional theory based calculation of the ground state structure of GFO along with experimental determination of the structural parameters of a polycrystalline sample at RT. The calculations confirm that the ground state structure of GFO is A-type antiferromagnetic. We find that the $\mathrm{Ga} / \mathrm{Fe}-\mathrm{O}$ bonds have a largely ionic character with no anomaly in the magnitude of corresponding Born effective charges. The calculations indicate the presence of a large spontaneous polarization $\left(P_{\mathrm{s}}\right)$ in $\mathrm{GFO}$ with a magnitude of $\sim 59 \mu \mathrm{C} \mathrm{cm}^{-2}$ along its $b$-axis.

\section{Calculation and experimental details}

Our entire calculation is based on the first-principles density functional theory [14]. The Vienna $a b$ initio simulation package (VASP) [15, 16] was used with the projector augmented wave method (PAW) [17]. The Kohn-Sham equation [18] was solved using the local spin density approximation (LSDA $+U$ ) [19] with the Hubbard parameter, $U=5 \mathrm{eV}$, and the exchange interaction, $J=1 \mathrm{eV}$. LSDA $+U$ has been found to be quite efficient in describing strongly correlated multiferroic systems $[20,21]$ in comparison to the conventional local density approximation (LDA) and generalized gradient approximation (GGA). We employed the simplified, rotationally invariant approach introduced by Dudarev [22]. The value of $U$ was optimized such that the moments of the magnetic ions are satisfactorily described with respect to the experiment [1]. We also checked that a small variation of $U$ from the optimized value does not alter the structural stability.

The calculations are based on the stoichiometric GFO assuming no partial occupancies of the constituent ions. We included three valence electrons for $\mathrm{Ga}\left(4 \mathrm{~s}^{2} 4 \mathrm{p}^{1}\right)$, eight for $\mathrm{Fe}\left(3 \mathrm{~d}^{7} 4 s^{1}\right)$ and six for $\mathrm{O}\left(2 \mathrm{~s}^{2} 2 \mathrm{p}^{4}\right)$ ions. A plane wave energy cutoff of $550 \mathrm{eV}$ was used. The conjugate gradient algorithm [23] was used for the optimization of the structure. All the calculations were performed at $0 \mathrm{~K}$. Structural optimization and calculation of the electronic band structure and density of states were carried out using a MonkhorstPack [24] $7 \times 7 \times 12$ mesh. Born effective charges, and spontaneous polarization for the ground state structure were calculated using the Berry phase method [25] with a $3 \times$ $3 \times 3$ mesh. A comparison of some of the results of the $3 \times 3 \times 3$ mesh with those obtained using a denser $k$ mesh shows good agreement. We also repeated some of our calculations using the generalized gradient approximation
$(\mathrm{GGA}+U)$ with the optimized version of the Perdew-BurkeErnzerhof functional for solids (PBEsol) [26] to check the consistency of LSDA $+U$ calculations. The effect of the Ga $3 \mathrm{~d}$ semicore state was studied with LSDA $+U$ and GGA $+U$ methods using a different pseudopotential of Ga that includes 13 valence electrons $\left(3 d^{10} 4 s^{2} 4 p^{1}\right)$, while keeping all other pseudopotentials the same. The calculations were performed using a Monkhorst-Pack $3 \times 3 \times 3$ mesh. We started our calculations with the experimental structural parameters obtained from the neutron diffraction spectra of crushed single crystals of $\mathrm{GaFeO}_{3}$ obtained at $4 \mathrm{~K}$ [1]. In order to obtain the ground state structure, the ionic positions, lattice parameters and unit-cell shape were sequentially relaxed in such a way that the pressure on the optimized structure was almost zero and the Hellmann-Feynman forces were less than $0.001 \mathrm{eV} \AA^{-1}$.

The Born effective charge (BEC) tensor of an atom $k$, is defined as:

$$
Z_{k, \gamma \alpha}^{*}=V \frac{\delta P_{\gamma}}{\delta \tau_{k, \alpha}}=\frac{\delta F_{k, \alpha}}{\delta \xi_{\gamma}}=-\frac{\partial^{2} E}{\partial \xi_{\gamma} \partial \tau_{k, \alpha}}
$$

where $P_{\gamma}$ represents the polarization induced by the periodic displacement $\tau_{k, \alpha}$ or by the force $F_{k, \alpha}$ induced by an electric field $\xi_{\gamma} . \quad E$ is the total energy of the unit cell. In the present calculation we displaced each ion by a small but finite distance along the three right handed Cartesian axes (unit-cell parameters are along the Cartesian axes) one at a time and calculated the polarization. The change in polarization with respect to the undistorted structure divided by the displacement gives the elements of Born charges in a particular direction for an ion.

To corroborate the calculations with the experimental data, we synthesized a polycrystalline $\mathrm{GaFeO}_{3}(\mathrm{Ga}: \mathrm{Fe}-1: 1)$ sample using the conventional solid-state-reaction route by mixing $\beta-\mathrm{Ga}_{2} \mathrm{O}_{3}$ and $\alpha-\mathrm{Fe}_{2} \mathrm{O}_{3}$ powders. The powder diffraction data of the sintered pellet were collected on a Philips X'Pert Pro MRD diffractometer using $\mathrm{Cu} \mathrm{K} \alpha$ radiation. Further, Rietveld refinement of the data was done using the FULLPROF 2000 [27] package using orthorhombic $P c 2_{1} n$ symmetry of GFO.

\section{Results and discussion}

\subsection{Structural optimization: ground state structure}

To determine the ground state structure as well as to elucidate the magnetic structure of GFO, we considered four possible antiferromagnetic spin configurations as shown in figures 1(a)-(d), i.e. AFM-1 (A-type), AFM-2 (C-type), AFM-3 (G-type) and AFM-4. It should be noted that AFM-4 represents a possible spin configuration which is different from the conventional A, C and G-type. In addition to the above, we also considered other possible spin configurations which would ensure antiferromagnetism in GFO, but were found to be equivalent to one of the above shown in figures 1(a)-(d). While previous reports confirm the ground state structure of GFO to be antiferromagnetic [13], there is no discussion on the possible antiferromagnetic spin configurations. The results of the total energy calculations of 


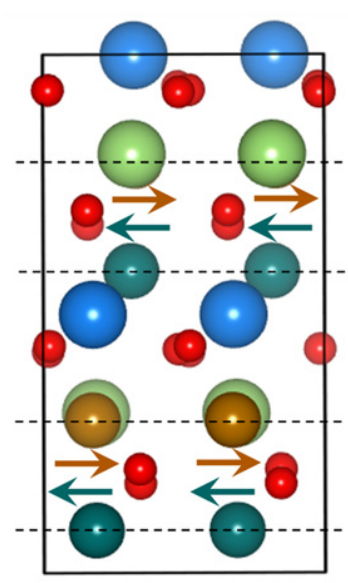

(a)

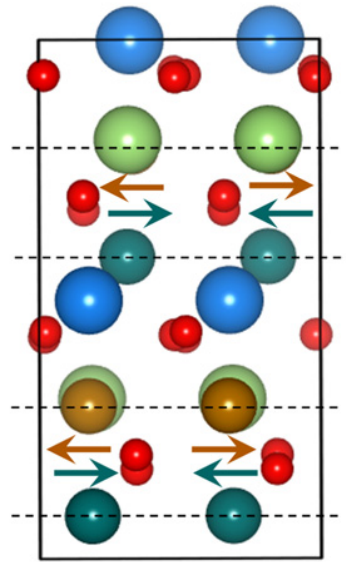

(c)

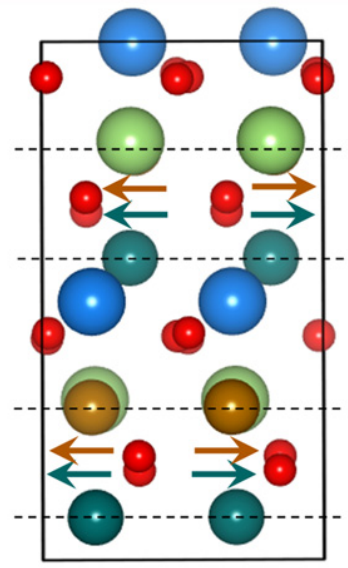

(b)

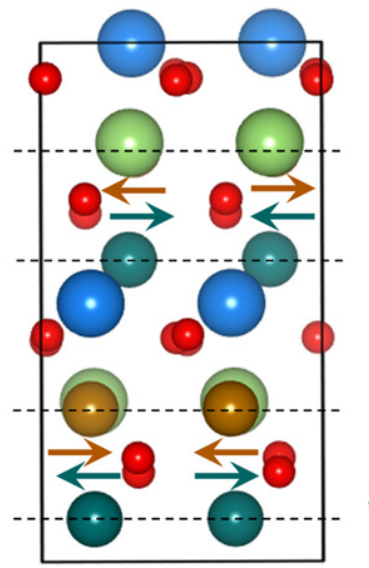

(d)

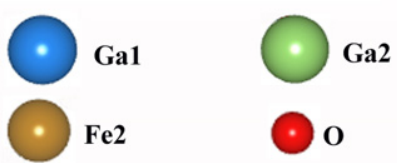

Figure 1. Schematics of different antiferromagnetic spin configurations considered in the present calculations. The configurations are assigned as (a) AFM-1 (A-type), (b) AFM-2 (C-type), (c) AFM-3 (G-type) and (d) AFM-4 (different variant).

the four structures show that while the energies of the AFM-3 and AFM-4 structures are maximum (947.202 meV/unit-cell and $839.823 \mathrm{meV} /$ unit-cell, respectively higher than AFM1 structure), the AFM-2 falls in the intermediate range with the AFM-1 having the lowest energy. Hence, the stability of different spin configurations can be ordered as AFM-1 > AFM-2 > AFM-4 > AFM-3. On this basis, we can conclude that the AFM-1 spin configuration is the most favored configuration in the $P c 2{ }_{1} n$ symmetry of GFO in the ground state. Hence, all further calculations were performed on the AFM-1 structure.

The ground state crystal structure was determined by further relaxing the size, shape and ionic positions while maintaining the AFM-1 spin configuration. The calculations show that the ground state structure retains the original $P c 2{ }_{1} n$ symmetry observed experimentally at $298 \mathrm{~K}$ [8] and at $4 \mathrm{~K}$ [1] and also corroborated by our x-ray diffraction (XRD) data (shown in figure 2). A schematic representation of the ground

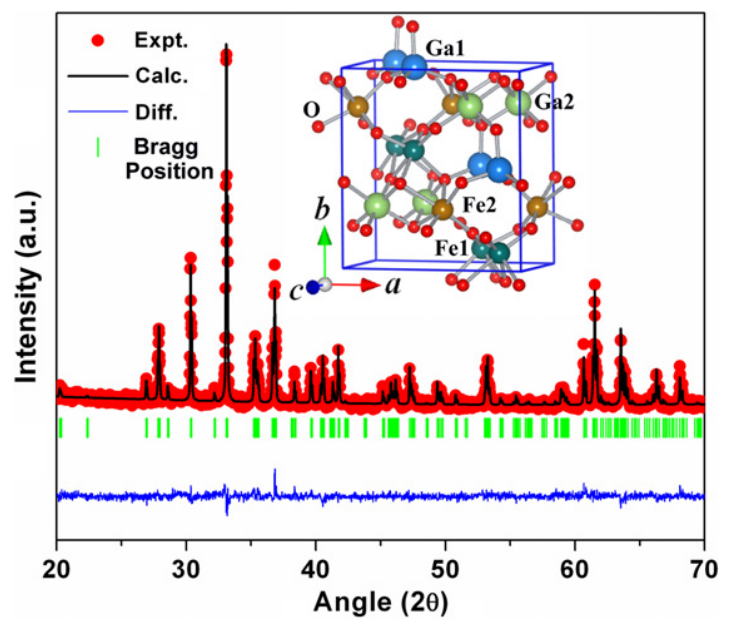

Figure 2. Rietveld refinement of RT XRD data of stoichiometric GFO. The inset shows a schematic of the crystal structure of GFO having orthorhombic $P c 2_{1} n$ symmetry.

state crystal structure is shown in the inset of figure 2. The calculated ground state lattice parameters, using LSDA $+U$, are $a=8.6717 \AA, b=9.3027 \AA$ and $c=5.0403 \AA$ which correspond well with our experimental data: $a=$ $8.7345 \AA, b=9.3816 \AA$ and $c=5.0766 \AA$. Our calculation using the GGA $+U$ method yielded the ground state lattice parameters as follows: $a=8.77119 \AA, b=9.40936 \AA$ and $c=5.09811 \AA$. The calculated and experimentally determined lattice parameters are also in close agreement with the previously reported data: $a=8.71932 \AA, b=$ $9.36838 \AA$ and $c=5.06723 \AA$ at $4 \mathrm{~K}[1], a=8.72569 \AA$, $b=9.37209 \AA$ and $c=5.07082 \AA$ at $230 \mathrm{~K}$ [1], $a=8.7512 \AA, b=9.3993 \AA$ and $c=5.0806 \AA$ at $298 \mathrm{~K}$ [8]. Thus, the lattice parameters calculated using $\mathrm{GGA}+U$ and LSDA $+U$ at $0 \mathrm{~K}$ are in good agreement with the experimental data obtained at $4 \mathrm{~K}$ [1], within a difference of $\sim \pm 7 \%$. This difference can be attributed to the approximation schemes of LSDA and GGA. Moreover, it should be noted that the calculated ground state structure is perfectly ordered while the experimental structures may consist of partial cation site occupancies. Many first-principles calculations on $\mathrm{Ga}$ containing oxides include $\mathrm{Ga} 3 \mathrm{~d}$ as semicore states [28]. To investigate the effect of the $\mathrm{Ga} 3 \mathrm{~d}$ semicore state, we also performed structural optimization of GFO using LSDA $+U$ and GGA $+U$ with a different pseudopotential of $\mathrm{Ga}$ that includes 13 valence electrons $\left(3 \mathrm{~d}^{10} 4 \mathrm{~s}^{2} 4 \mathrm{p}^{1}\right)$, while keeping all other pseudopotentials the same. Structural optimization showed that the optimized lattice parameters are $a=8.642695 \AA$, $b=9.271509 \AA$ and $c=5.023425 \AA$ for LSDA $+U$, and $a=8.836875 \AA, b=9.479817 \AA$ and $c=5.136288 \AA$ for $\mathrm{GGA}+U$. A comparison of these values with the experimental data as shown above, shows these to be even farther from the experimental data, while a comparison with the values calculated without considering Ga $3 \mathrm{~d}$ semicore state shows that inclusion of $\mathrm{Ga} 3 \mathrm{~d}$ semicore state slightly underestimates the lattice parameters in LSDA $+U$ but overestimates them in $\mathrm{GGA}+U$. We therefore performed further calculations using the pseudopotential of $\mathrm{Ga}$ that includes three valence electrons 
Table 1. Calculated ground state ionic positions of orthorhombic $\left(P c 2{ }_{1} n\right)$ GFO using LSDA $+U$ and GGA $+U$ along with Rietveld refined experimental data.

\begin{tabular}{|c|c|c|c|c|c|c|c|c|c|}
\hline \multirow[b]{2}{*}{ Ion } & \multicolumn{3}{|c|}{$\mathrm{LSDA}+U$} & \multicolumn{3}{|c|}{$\mathrm{GGA}+U$} & \multicolumn{3}{|c|}{ Experiment at $298 \mathrm{~K}$} \\
\hline & $x$ & $y$ & $z$ & $x$ & $y$ & $z$ & $x$ & $y$ & $z$ \\
\hline a1 (4a) & 0.15101 & 0.99844 & 0.17665 & 0.15125 & 0.99844 & 0.175969 & 0.15291 & 0.00000 & 0.17900 \\
\hline $\mathrm{Ga} 2(4 \mathrm{a})$ & 0.16068 & 0.30818 & 0.81637 & 0.16087 & 0.30817 & 0.81653 & 0.15902 & 0.30413 & 0.81446 \\
\hline Fe1 (4a) & 0.15512 & 0.58224 & 0.18817 & 0.15477 & 0.58248 & 0.18690 & 0.15299 & 0.58079 & 0.20291 \\
\hline $\mathrm{Fe} 2(4 \mathrm{a})$ & 0.03075 & 0.79453 & 0.67380 & 0.03078 & 0.79453 & 0.67314 & 0.03197 & 0.79907 & 0.67050 \\
\hline $\mathrm{O} 1(4 \mathrm{a})$ & 0.32292 & 0.42757 & 0.98443 & 0.32260 & 0.42709 & 0.98386 & 0.32120 & 0.42638 & 0.98250 \\
\hline $\mathrm{O} 2(4 \mathrm{a})$ & 0.48576 & 0.43140 & 0.51922 & 0.48600 & 0.43128 & 0.51976 & 0.98915 & 0.43217 & 0.51623 \\
\hline O3 (4a) & 0.99672 & 0.20019 & 0.65659 & 0.99694 & 0.20084 & 0.65734 & 0.99730 & 0.19794 & 0.66331 \\
\hline O4 (4a) & 0.16218 & 0.19907 & 0.15803 & 0.16176 & 0.19902 & 0.15796 & 0.16015 & 0.19924 & 0.14523 \\
\hline O5 (4a) & 0.16719 & 0.67266 & 0.84410 & 0.16752 & 0.67224 & 0.84306 & 0.15901 & 0.66492 & 0.84351 \\
\hline O6 (4a) & 0.16636 & 0.93781 & 0.52144 & 0.16635 & 0.93800 & 0.52079 & 0.16260 & 0.94593 & 0.52414 \\
\hline
\end{tabular}

Table 2. Calculated bond lengths from the ground state ionic positions of orthorhombic $\left(P c 2{ }_{1} n\right)$ GFO along with experimental data from the present work and previously reported data.

\begin{tabular}{|c|c|c|c|c|c|c|}
\hline \multirow[b]{2}{*}{ Bond length $(\AA)$} & \multicolumn{2}{|c|}{ Theory } & \multicolumn{3}{|c|}{ Experimental data } & \multirow{2}{*}{$\begin{array}{l}\text { \%Difference (LSDA }+U- \\
\text { experiment at } 4 \mathrm{~K})\end{array}$} \\
\hline & $\mathrm{LSDA}+U$ & $\mathrm{GGA}+U$ & $298 \mathrm{~K}^{\mathrm{a}}$ & $4 \mathrm{~K}^{\mathrm{b}}$ & $298 \mathrm{~K}^{\mathrm{c}}$ & \\
\hline $\mathrm{Ga} 1-\mathrm{O} 2$ & 1.849 & 1.869 & 1.853 & 1.844 & 1.851 & 0.27 \\
\hline Ga1-O6 & 1.832 & 1.852 & 1.826 & 1.822 & 1.813 & 0.55 \\
\hline Ga1-O6' & 1.854 & 1.873 & 1.863 & 1.836 & 1.867 & 0.98 \\
\hline Ga1-O4 & 1.871 & 1.892 & 1.878 & 1.857 & 1.852 & 0.75 \\
\hline $\mathrm{Ga} 2-\mathrm{O} 3$ & 1.918 & 1.935 & 1.891 & 1.892 & 1.927 & 1.37 \\
\hline Ga2-O1 & 1.983 & 1.998 & 2.012 & 1.985 & 2.011 & -0.10 \\
\hline $\mathrm{Ga} 2-\mathrm{O} 2$ & 1.993 & 2.019 & 2.041 & 2.006 & 2.054 & -0.65 \\
\hline Ga2-O4 & 2.007 & 2.032 & 2.050 & 2.059 & 2.077 & -2.53 \\
\hline $\mathrm{Ga} 2-\mathrm{O} 4^{\prime}$ & 1.999 & 2.021 & 1.946 & 1.996 & 2.037 & 0.15 \\
\hline $\mathrm{Ga} 2-\mathrm{O} 1^{\prime}$ & 2.013 & 2.037 & 2.046 & 2.053 & 2.051 & -1.95 \\
\hline $\mathrm{Fe} 1-\mathrm{O} 1$ & 2.082 & 2.114 & 2.041 & 2.064 & 2.058 & 0.87 \\
\hline $\mathrm{Fe} 1-\mathrm{O} 1^{\prime}$ & 2.291 & 2.319 & 2.347 & 2.354 & 2.361 & -2.68 \\
\hline $\mathrm{Fe} 1-\mathrm{O} 2$ & 2.046 & 2.068 & 2.094 & 2.074 & 2.06 & -1.35 \\
\hline $\mathrm{Fe} 1-\mathrm{O} 3$ & 1.884 & 1.908 & 1.842 & 1.905 & 1.866 & -1.10 \\
\hline $\mathrm{Fe} 1-\mathrm{O} 5$ & 1.923 & 1.943 & 1.957 & 1.918 & 1.936 & 0.26 \\
\hline $\mathrm{Fe} 1-\mathrm{O}^{\prime}$ & 1.930 & 1.949 & 1.989 & 1.934 & 1.934 & -0.21 \\
\hline $\mathrm{Fe} 2-\mathrm{O} 1$ & 2.326 & 2.352 & 2.328 & 2.324 & 2.354 & 0.09 \\
\hline $\mathrm{Fe} 2-\mathrm{O} 2$ & 2.042 & 2.064 & 2.056 & 2.025 & 2.064 & 0.84 \\
\hline $\mathrm{Fe} 2-\mathrm{O} 4$ & 2.075 & 2.098 & 2.137 & 2.131 & 2.093 & -2.63 \\
\hline $\mathrm{Fe} 2-\mathrm{O} 3$ & 1.897 & 1.917 & 1.959 & 1.943 & 1.946 & -2.37 \\
\hline $\mathrm{Fe} 2-\mathrm{O} 5$ & 1.850 & 1.874 & 1.894 & 1.875 & 1.872 & -1.33 \\
\hline $\mathrm{Fe} 2-\mathrm{O} 6$ & 1.936 & 1.959 & 1.937 & 1.958 & 1.971 & -1.12 \\
\hline $\mathrm{Fe} 1-\mathrm{Fe} 2$ & 3.201 & 3.240 & 3.164 & 3.201 & 3.234 & 0 \\
\hline $\mathrm{Ga} 1-\mathrm{Ga} 2$ & 3.231 & 3.271 & 3.286 & 3.246 & - & -0.46 \\
\hline $\mathrm{Fe} 2-\mathrm{Ga} 2$ & 3.062 & 3.100 & 3.102 & 3.089 & 3.007 & -0.87 \\
\hline Fe1-Ga1 & 3.320 & 3.354 & 3.387 & 3.328 & - & -0.24 \\
\hline $\mathrm{Fe} 1-\mathrm{Ga} 2$ & 3.165 & 3.198 & 3.123 & 3.216 & 3.121 & -1.59 \\
\hline
\end{tabular}

${ }^{\mathrm{a}}$ Present work. ${ }^{\mathrm{b}}$ Reference [1]. ${ }^{\mathrm{c}}$ Reference [7].

$\left(4 s^{2} 4 p^{1}\right)$ since it provides a better accuracy of the structural parameters.

The present experimentally determined ionic positions of stoichiometric GFO, along with the calculated ground state ionic positions are listed in table 1 which shows that $\mathrm{Fe} 1$ and $\mathrm{Fe} 2$ ions lie on alternate planes parallel to the $a c$-plane. Since $\mathrm{Fe} 1$ and Fe 2 have anti-parallel spin configurations and are situated on alternate parallel planes, we conclude (see figure 1) that the ground state magnetic structure of GFO is A-type antiferromagnetic. Figure 2 (inset) also shows the coordination of the cations by oxygen: Ga1 is tetrahedrally coordinated while Ga2, Fe1 and Fe 2 are octahedrally coordinated by the surrounding oxygen atoms.

From the positions of the ions in the calculated ground state structures and in the experimentally determined stoichiometric GFO at $298 \mathrm{~K}$, we calculated the bond lengths of cations with neighboring oxygen ions. Table 2 consisting of calculated cation-oxygen and cation-cation bond lengths, shows a good agreement with the present and previous XRD [8] and neutron data [1]. Minor differences can be attributed to a number of factors, such as temperature, site disorder and the limitation of the exchange correlation 


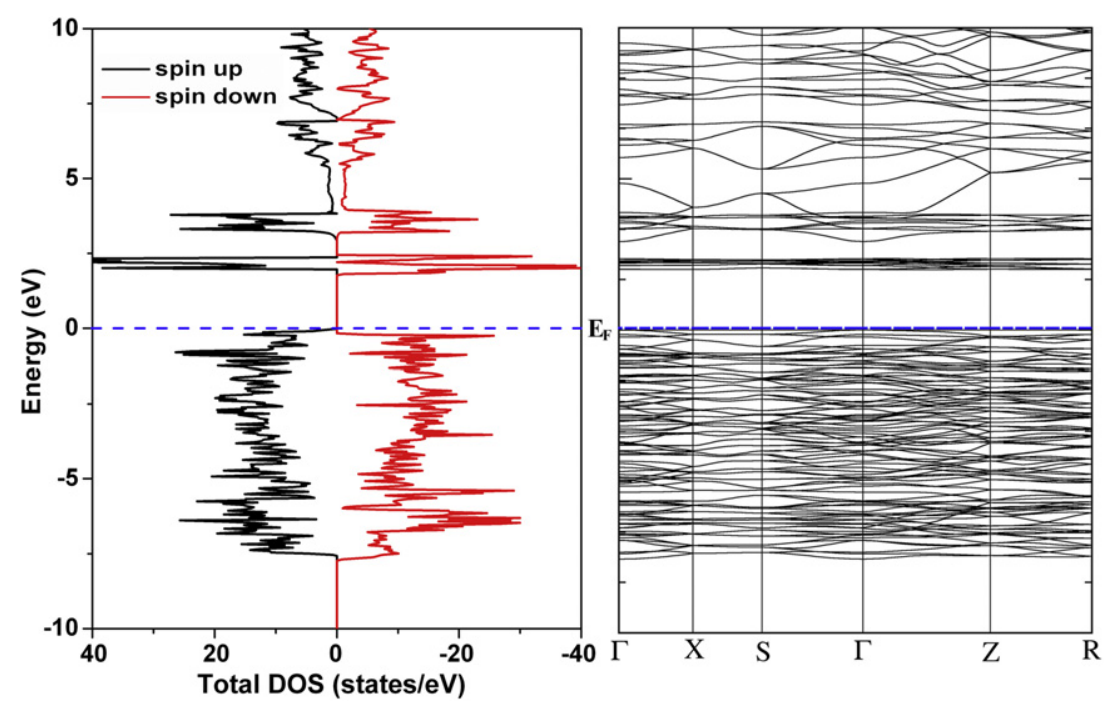

Figure 3. Electronic structures of orthorhombic $\left(P c 2{ }_{1} n\right) \mathrm{GaFeO}_{3}$ calculated using the LSDA $+U$ method. The left panel shows a plot of the total density of states as a function of energy while the right panel shows the electronic band structure along high symmetry directions. The zero in the energy axis is set at the highest occupied level.

functionals used in our study. Using the bond length data from table 2, we also calculated the structural distortions of the oxygen polyhedra [29]. The distortion can be quantified by determining the distortion index [30] which is defined as:

$$
\mathrm{DI}=\frac{1}{n} \sum_{i=1}^{n} \frac{\left(l_{i}-l_{\mathrm{av}}\right)}{l_{\mathrm{av}}}
$$

where $l_{i}$ is the bond length of $i$ th coordinating ion and $l_{\mathrm{av}}$ is the average bond length.

The calculations show that the DI values of Ga1-O tetrahedron are $\sim 0.006$ (at ground state for both LSDA $+U$ and GGA $+U$ ) and 0.008 at $298 \mathrm{~K}$ and as a result, the effective anion coordination $(\sim 3.99$ (LSDA $+U$ and GGA $+U), \sim 3.98$ (expt.)) is almost identical to that of a regular tetrahedron, i.e. 4. Here, the effective coordination number (ECoN) [31] is defined as:

$$
\mathrm{ECoN}=\sum_{i} \exp \left\{1-\left(\frac{l_{i}}{l_{\mathrm{av}}}\right)^{6}\right\} .
$$

In contrast, the Ga2-O octahedron shows appreciable distortion $(\mathrm{DI} \sim 0.012(\mathrm{LSDA}+U), \sim 0.013(\mathrm{GGA}+U)$ and $\sim 0.026$ (expt.)) compared to a regular octahedron which is also reflected in a smaller coordination number of 5.93 $(\mathrm{LSDA}+U), 5.92(\mathrm{GGA}+U)$ and 5.75 (expt.) than the perfect octahedral coordination, i.e. 6. This distortion is more significant in the case of $\mathrm{Fe} 1-\mathrm{O}$ and $\mathrm{Fe} 2-\mathrm{O}$ octahedra with DI values of $0.056(\mathrm{LSDA}+U), 0.057(\mathrm{GGA}+U)$ and 0.057 (expt.), and $0.063(\mathrm{LSDA}+U), 0.062(\mathrm{GGA}+U)$ and 0.059 (expt.), respectively, while the corresponding average coordination numbers are $5.05(\mathrm{LSDA}+U), 5.04(\mathrm{GGA}+U)$ and 4.74 (expt.), and 4.81 (LSDA $+U), 4.83(\mathrm{GGA}+U)$ and 4.92 (expt.), respectively. Thus, it is observed that for almost all the oxygen polyhedra, the cations are displaced from the center of the polyhedra. The significance of these distortions lies in imparting the non-centrosymmetry to the structure which results in the development of spontaneous polarization in GFO, as shown later in section 3.3.

\subsection{Electronic band structure, density of states and bonding}

Figure 3 shows the LSDA $+U$ calculated electronic band structure along the high symmetry directions and the total density of states of GFO. The Fermi energy is fixed at $0 \mathrm{eV}$. The figure shows the plots of the band structure and total density of states demonstrating that the bands are spread over three major energy windows. The uppermost part of the valence band spreads over $-7.73-0 \mathrm{eV}$. Above the Fermi level, the conduction band can again be divided into two parts: the first part is in the energy range from 1.77 to $2.45 \mathrm{eV}$ while another part is in the energy range from 3.0 to $16.83 \mathrm{eV}$ (shown partially). The angular momentum character of the bands spread over different energy regions can be determined from the partial density of states (PDOS) of the constituent ions. The PDOS of Fe1, Ga1 and $\mathrm{O} 1$ ions are shown in figure 4. As the nature of the PDOS of the other ions is similar, these plots are not shown here. These figures show that the valence band $(-7.73-0 \mathrm{eV})$ mainly consists of $\mathrm{Fe} 3 \mathrm{~d}$ and $\mathrm{O} 2 \mathrm{p}$ states with a significant amount of $\mathrm{Ga} 4 \mathrm{~s}$ and $\mathrm{Ga} 4 \mathrm{p}$ characters also present in the lower energy side of this energy range. Beyond the Fermi level, a narrow energy band (1.77-2.45 eV) contains mainly Fe $3 \mathrm{~d}$ character. The highest energy window (3.0$16.83 \mathrm{eV}$ ) has contributions from Fe 3d, Ga 4s, Ga 4p and O 2p states. More importantly, the PDOS demonstrates significant hybridization of $\mathrm{Fe} 3 \mathrm{~d}, \mathrm{Ga} 4 \mathrm{p}$ and $\mathrm{O} 2 \mathrm{p}$ states throughout the uppermost part of the valence band. Such hybridization of the transition metal $\mathrm{d}$ state and $\mathrm{O} 2 \mathrm{p}$ state has been found to impart ferroelectricity in a number of perovskite oxides [32, 33] and can be of interest in GFO too.

As shown in figure 3, our LSDA $+U$ calculations yielded a direct band gap $\left(E_{\mathrm{g}}\right)$ of $\sim 2.0 \mathrm{eV}(\Gamma-\Gamma)$ while $\mathrm{GGA}+U$ calculations showed a direct band gap of $\sim 2.25 \mathrm{eV}$. 


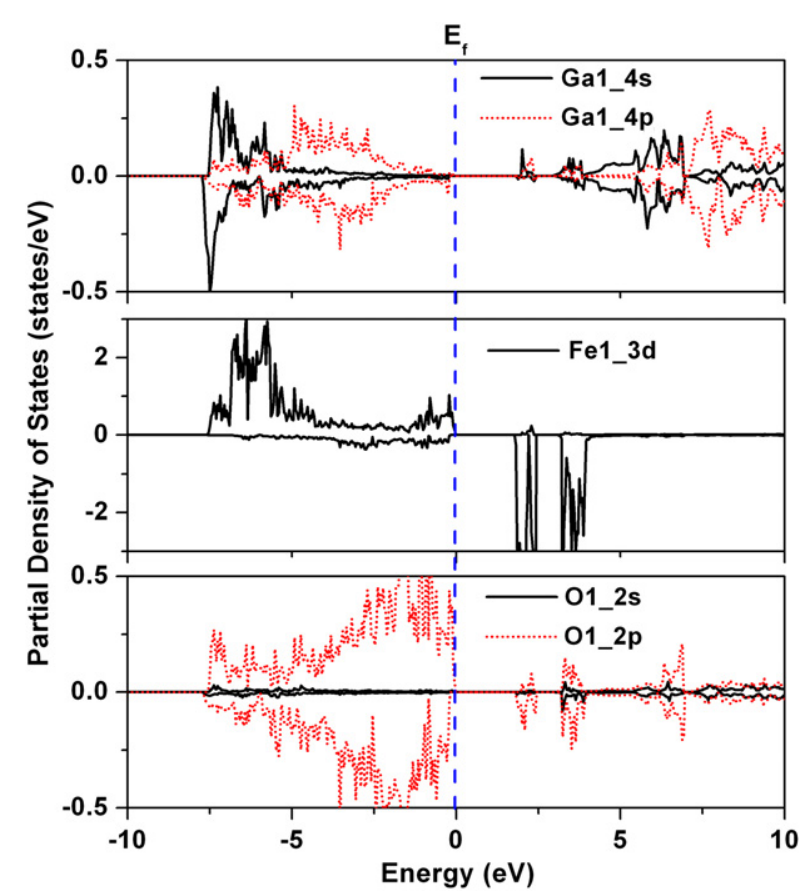

Figure 4. PDOS plots of Ga1 $4 \mathrm{~s}$ and $4 \mathrm{p}$ states, Fe1 $3 \mathrm{~d}$ state and $\mathrm{O} 12 \mathrm{~s}$ and $2 \mathrm{p}$ states calculated using the LSDA $+U$ method. The vertical blue line indicates the Fermi level.

Calculation of the band structure using the LSDA $+U$ method with pseudopotential treating $\mathrm{Ga} 3 \mathrm{~d}$ as a semicore state did not reveal any noticeable change from that of our earlier calculation and a direct band gap $\left(E_{\mathrm{g}}\right)$ of $\sim 1.98 \mathrm{eV}(\Gamma-\Gamma)$ was obtained. However, experimental studies based on the optical absorption spectra of GFO report a band gap of 2.7$3.0 \mathrm{eV}$. [34] The difference between the calculated band gap and the experimental data is expected (due to underestimation of band gap by the LSDA and GGA methods) and is common in the electronic structure calculation of oxides [35, 36].

Moreover, the PDOS data in figure 4 can also shed light on the bonding behavior in GFO, especially the partial covalency of cation-anion bonds, which can be further correlated with the functional properties of GFO. From figure 4, we find that Fe 3d and $\mathrm{O} 2 \mathrm{p}$ states are significantly hybridized in the uppermost part of the valence band in GFO. For a detailed analysis, we have plotted the charge density distribution calculated using LSDA $+U$, on three principal planes of the unit cell as shown in figure 5(a). The figure shows that although most of the charges are symmetrically distributed along the radius of the circles, indicating the largely ionic nature of bonding, a small amount of covalency is shown by minor asymmetry of the charges around $\mathrm{O}$ ions connected to the $\mathrm{Fe} 1, \mathrm{Fe} 2, \mathrm{Ga} 1$ and $\mathrm{Ga} 2$ ions.

However, the nature of binding interaction as determined from the charge density distribution alone is not conclusive. We therefore utilized the electron localization function (ELF) which provides a measure of the local influence of the Pauli repulsion on the behavior of the electrons and allows the mapping of core, bonding and nonbonding regions of the crystal in real space. Thus the ELF can be used as a tool to differentiate the nature of different types of bonds [37]. A large value of the ELF indicates a region of small Pauli repulsion, in other words, a space with anti-parallel spin configuration while the position with the maximum ELF value has the signature of an electron pair [37]. Figures 5(b) and (c) show the ELF distribution in three principal planes and in the entire unit cell of GFO, respectively, calculated by the LSDA $+U$ method. Figure 5(b) also depicts the maximum ELF value at $\mathrm{O}$ sites and small values at the $\mathrm{Fe}$ and $\mathrm{Ga}$ sites indicating a charge transfer interaction from the $\mathrm{Fe} / \mathrm{Ga}$ to the $\mathrm{O}$ sites. Comparing figures 5(a) and (b), we find that an almost complete charge transfer takes place between the $\mathrm{Fe} 2$ and $\mathrm{O} 3$ ions. A similar charge transfer, albeit to a lesser extent, is also observed between the $\mathrm{Fe} 1$ and $\mathrm{O} 1, \mathrm{O} 2$ ions. Thus we can conclude that the $\mathrm{Fe}-\mathrm{O}$ bonds in GFO are mostly ionic. In contrast, polarization of the ELF from $\mathrm{O}$ sites toward other $\mathrm{O}$ sites and the finite value of the ELF between $\mathrm{O}$ and Ga1 (figure 5(b)) indicates some degree of covalent characteristics. A similar feature is expected for $\mathrm{Ga} 2-\mathrm{O}$ bonds as shown in figure 5(c). Therefore, from the charge density and ELF plots, we can assert that $\mathrm{Ga} / \mathrm{Fe}-\mathrm{O}$ bonds in $\mathrm{GFO}$ are largely of ionic

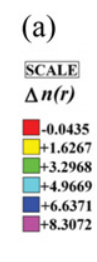

$$
\text { (b) }
$$

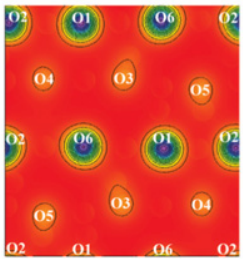

(001)

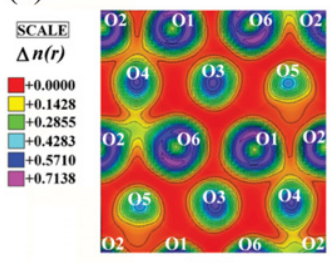

(001)

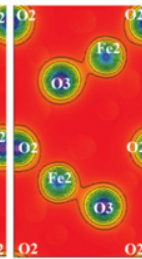

(100)

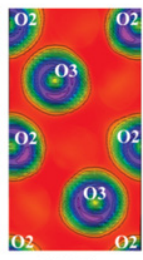

(100)
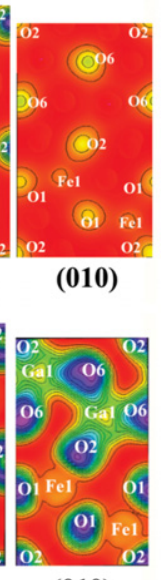

(010) (c) Ga1

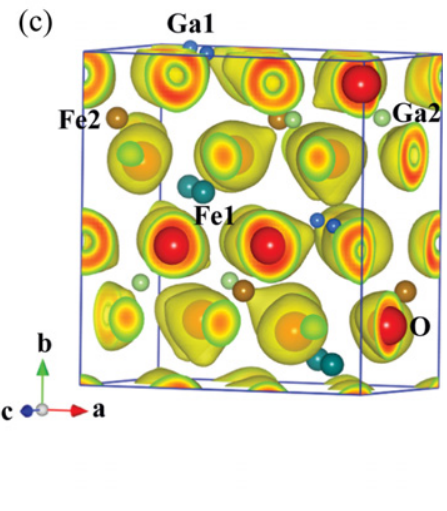

Figure 5. Plots of (a) charge density along three principal planes of GFO unit cell, (b) ELF calculated using the LSDA $+U$ method along three principal planes of GFO unit cell keeping the area of the planes in accordance with the respective lattice parameters and (c) 3D image of ELF distribution in the GFO unit cell. 
Table 3. Diagonal elements of the BEC tensors computed using the Berry phase technique within LSDA $+U$. The bond valence charges $(V)$ were calculated using bond length data based on the ground state structural parameters. Nominal ionic charges are also provided for comparison.

\begin{tabular}{|c|c|c|c|c|c|c|}
\hline \multirow[b]{2}{*}{ Ion } & \multirow{2}{*}{$\begin{array}{l}\text { Nominal ionic } \\
\text { charge }(e)\end{array}$} & \multicolumn{3}{|c|}{$Z^{*}(e)$} & \multirow{2}{*}{$\begin{array}{l}\text { Charge } \\
\text { distribution }(e)\end{array}$} & \multirow{2}{*}{$\begin{array}{l}\text { Bond valence } \\
\text { charge }(e)\end{array}$} \\
\hline & & $Z_{x x}$ & $Z_{y y}$ & $Z_{z z}$ & & \\
\hline $\mathrm{Ga} 1$ & 3 & 3.01 & 3.11 & 2.99 & 2.83 & 2.88 \\
\hline Ga2 & 3 & 3.57 & 3.16 & 3.53 & 3.23 & 3.02 \\
\hline $\mathrm{Fe} 1$ & 3 & 3.66 & 3.78 & 4.08 & 3.04 & 3.10 \\
\hline $\mathrm{Fe} 2$ & 3 & 3.68 & 3.38 & 3.82 & 2.90 & 3.20 \\
\hline O1 & -2 & -2.29 & -2.58 & -2.79 & -1.56 & - \\
\hline $\mathrm{O} 2$ & -2 & -2.45 & -2.29 & -2.41 & -2.12 & - \\
\hline $\mathrm{O} 3$ & -2 & -2.54 & -2.30 & -2.75 & -2.04 & - \\
\hline O4 & -2 & -2.27 & -2.85 & -2.17 & -2.02 & - \\
\hline O5 & -2 & -2.50 & -2.16 & -2.79 & -2.10 & - \\
\hline O6 & -2 & -2.32 & -2.08 & -2.40 & -2.16 & - \\
\hline
\end{tabular}

character. The ionicity is greater for $\mathrm{Fe}-\mathrm{O}$ bonds, while some degree of hybridization is observed in $\mathrm{Ga}-\mathrm{O}$ bonds indicating covalency.

\subsection{Born effective charge and spontaneous polarization}

The nature of bonding can further be correlated with the BECs $\left(Z^{*}\right)$, defined in section 2 . These charges are important quantities in elucidating the physical understanding of piezoelectric and ferroelectric properties since they describe the coupling between lattice displacements and the electric field. Born charges are also indicators of long range Coulomb interactions whose competition with the short range forces leads to the ferroelectric transition. Previous studies on many perovskite ferroelectrics show anomalously large Born charges for some of the ions [32, 33] which are often explained as a manifestation of the strong covalent character of the bonds between the specific ions. In GFO, from the charge density and ELF plots, we have observed that charge sharing between the $\mathrm{Ga} / \mathrm{Fe}$ and $\mathrm{O}$ ions in cation-oxygen bonds is not significant in comparison to conventional perovskite ferroelectrics $[32,33]$. On the other hand, from the structural data we find that the cation-oxygen polyhedra are highly distorted. Since ferroelectric and piezoelectric responses are combined manifestations of the structural distortions and effective charges of the constituent ions [38], it is imperative to calculate the BECs of the constituent ions in GFO. Such a calculation would help to elucidate the nature of cation-oxygen bonds and the origin of polarization in the material.

In the present work, we have calculated the BEC tensors of nonequivalent ions in the $P c 2_{1} n$ structure of GFO by slightly displacing each ion, one at a time, along three axes of the Cartesian coordinates and then calculating the resulting difference in polarization, using the Berry phase method [25]. We used the LSDA $+U$ technique for this calculation. Table 3 lists the three diagonal elements of the BEC tensors of each ion along with their nominal charges. Here, we observe that the Ga1 ion has elements of effective charge tensors close its nominal ionic charge and hence, we conclude that all the bonds between $\mathrm{Ga} 1$ and surrounding $\mathrm{O}$ ions are primarily ionic in nature. On the other hand, $\mathrm{Ga} 2$ develops a maximum effective charge of $3.53, \sim 18 \%$ higher with respect to its static charge of +3 . In contrast, both $\mathrm{Fe} 1$ and $\mathrm{Fe} 2$ ions show much higher increase in the effective charges, $36 \%$ and $27 \%$ respectively, while oxygen ions show a maximum reduction of $39.5 \%$ with respect to the nominal ionic charge. Interestingly, all these elements that have maximum change with respect to the respective static charges are along the $z$-axis (except for Ga1). However, the direction of $P_{\mathrm{s}}$ is along the $y$-axis, i.e. crystallographic $b$-direction [1]. Hence, unlike in most perovskite ferroelectrics [32, 33], the polarization in GFO is not due to large effective ionic charges. Instead, it is most likely to be caused by the structural distortion and noncentrosymmetry of the structure.

To compare our results on BECs with the effective charges calculated by other methods, we calculated these charges on each ionic site using the bond valence method in which the bond valence charge $(V)$ is defined as:

$$
V=\sum_{i} v_{i}=\sum_{i} \exp \left(\frac{R_{0}-R_{i}}{b}\right)
$$

where $R_{0}$ is the ideal bond length for a bond with valence 1 , $R_{i}$ is the measured bond length and $b$ is an empirical constant. We have also estimated the effective charge distribution [29] at different ionic sites based on the nominal ionic charges and polyhedra parameters. The results obtained from both methods are shown in table 3. Though these calculations are in no way comparable to the ab initio calculations, they are useful in getting a trend of the effective charges. The comparison shows that although the calculated BECs using the $a b$ initio method are larger than the effective charges calculated using the bond valence method and charge distribution method, all the calculations of effective charges point toward the fact that the cation-oxygen bonds in GFO are largely ionic and substantiate the discussion in the preceding paragraph.

The BECs can also be used to quantify the spontaneous polarization in GFO. Although previous studies $[1,12]$ indicate the direction of $P_{\mathrm{s}}$ along the [010]-direction, there is no conclusive experimental report on the value of $P_{\mathrm{s}}$. Although Arima et al [1] predicted a $P_{\mathrm{s}} \sim 2.5 \mu \mathrm{C} \mathrm{cm}^{-2}$ based on the displacement of $\mathrm{Fe}$ ions from the center of $\mathrm{FeO}_{6}$ octahedra, such a point charge calculation does not provide a correct estimate since various other contributions to $P_{\mathrm{s}}$ were neglected. 
As we see later, these other contributions are from sources such as $\mathrm{Ga} 1-\mathrm{O}$ tetrahedra and $\mathrm{Ga} 2-\mathrm{O}$ octahedra, and more importantly, effective ionic charges. To compare, we have calculated the $P_{\mathrm{s}}$ of GFO in its ground state using both nominal ionic charges and calculated BECs.

Further, from the crystallography perspective, GFO having a $P c 2{ }_{1} n$ space group allows the following symmetry operations to be performed to a point $(x, y, z)$ : (i) $c$-operation, a glide translation along half the lattice vector of the $c$-plane leading to $(1 / 2-x, y, 1 / 2+z)$, (ii) $2_{1}$ operation, twofold screw rotation around the $b$-axis leading to $(-x, 1 / 2+$ $y,-z)$ and (iii) $n$-operation, a glide translation along half of the face diagonal leading to $(1 / 2+x, 1 / 2+y, 1 / 2-z)$. Here, we observe that the application of the first and third operations ( $c$ and $n$ respectively) on the atom positions does not put any constraint on the displacement and in turn the polarization vector remains unrestricted. However, when a $2{ }_{1}$ symmetry operator is applied, i.e. when the cell is screw rotated by $180^{\circ}$ about the [010]-axis, i.e. the $b$-axis, it changes the crystal polarization from $\left(P_{x}, P_{y}, P_{z}\right)$ to $\left(-P_{x}, P_{y}\right.$, $\left.-P_{z}\right)$ as $(x, y, z)$ now becomes $(-x, y,-z)$. This clearly explains that the crystal polarization along the $a$ - and $c$-axes is equal to zero and is non-zero along the $b$-axis. Further, using the BECs from table 3, we calculated the spontaneous polarization $\left(P_{\mathrm{S}}\right)$ as $\sim 58.63 \mu \mathrm{C} \mathrm{cm} \mathrm{cm}^{-2}$ which is an order of magnitude larger than that predicted by Arima et al [1]. Similar calculation using the nominal ionic charges yielded $P_{\mathrm{s}}$ of $\sim 30.53 \mu \mathrm{C} \mathrm{cm}^{-2}$, almost half the value obtained using the BECs. We therefore conclude that though the values of Born charges of the constituent ions are not anomalously large unlike some perovskite ferroelectrics [32, 33], they do seem to affect the spontaneous polarization response in GFO rather significantly.

We also calculated the partial polarization in order to estimate the relative contribution of individual ions. A schematic of the partial polarization contributions from individual ions toward the total spontaneous polarization has been shown in figure 6 . It was found that while the contribution from Ga1 is the largest, it is counterbalanced by the opposite contributions from $\mathrm{Fe} 1, \mathrm{O} 1, \mathrm{O} 2$ and $\mathrm{O} 6$. Interestingly, the structure data (table 1 and figure 2) also show that these ions are the most asymmetrically placed around the inversion center of symmetry while the $\mathrm{Ga} 2$ and $\mathrm{Fe} 2$ cations maintain an almost centrosymmetric configuration and contribute least to the total polarization. Therefore, we conclude that the spontaneous polarization in GFO is primarily contributed by the asymmetrically placed $\mathrm{Ga} 1, \mathrm{Fe} 1, \mathrm{O} 1, \mathrm{O} 2$ and $\mathrm{O} 6$ ions. However, at elevated temperatures, site disordering between Fe1 and Ga1 sites is expected [1] which may substantially lower the spontaneous polarization. This should be of interest for further theoretical investigations incorporating the effect of disorder on calculations.

\section{Conclusions}

We have presented a theoretical study of the structure-property relationship in gallium ferrite, supported by experimental data. First-principles density functional theory based calculations

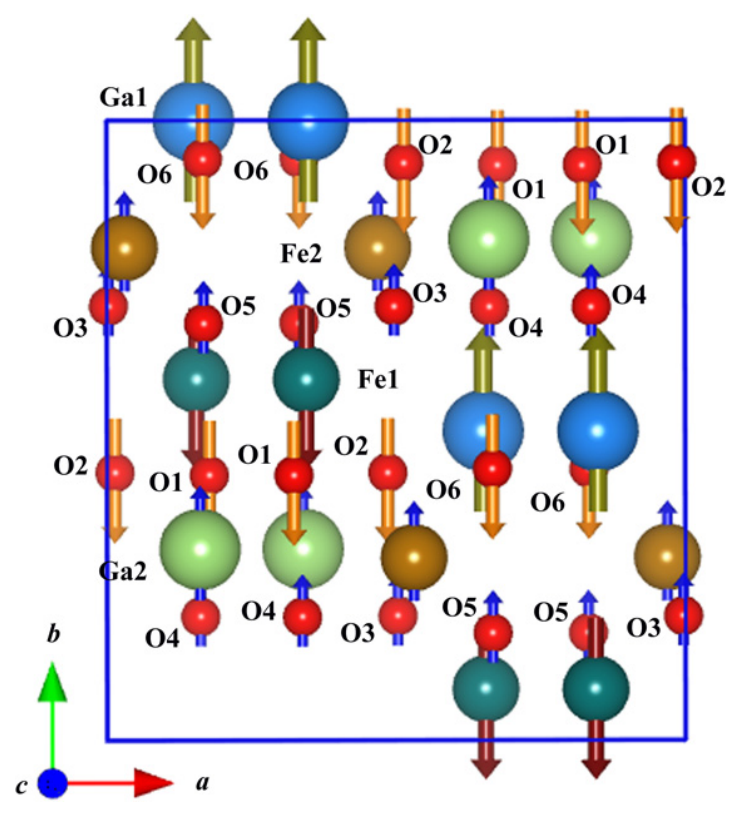

Figure 6. Schematic diagram showing partial polarization of individual ions along the crystallographic $b$-direction. The strength and direction of polarization is depicted by the size and direction of the arrows.

were performed to calculate the ground state structure of gallium ferrite. The calculations support an orthorhombic structure with $P c 2_{1} n$ symmetry and A-type antiferromagnetic spin configuration in the ground state with calculated ground state lattice parameters, bond strength and bond angles agreeing well with the experimental results. While the electronic density of states show hybridization among Fe 3d, $\mathrm{Ga} 4 \mathrm{~s}, \mathrm{Ga} 4 \mathrm{p}$ and $\mathrm{O} 2 \mathrm{p}$ states, calculations of electronic charge density demonstrate almost symmetrical charge distribution on most of the major planes indicating an ionic nature of bonds. Calculation of the electron localization function further supported a largely ionic character of $\mathrm{Fe}-\mathrm{O}$ bonds and a finite degree of hybridization among $\mathrm{O}, \mathrm{Ga} 1$ and Ga2 ions. Moreover, the lack of any significant anomaly in the Born effective charges with respect to the corresponding nominal ionic charges again emphasized the ionic character of the bonds. The calculations also showed a spontaneous polarization of $\sim 59 \mu \mathrm{C} \mathrm{cm}^{-2}$ along the $b$-direction, i.e. [010]axis, attributed primarily to the structural distortion.

\section{Acknowledgments}

The authors thank Professor M K Harbola (Physics, IIT Kanpur) for valuable discussions on the work and his suggestions. AR and SM thank the Ministry of Human Resources, Government of India for financial support.

\section{References}

[1] Arima T et al 2004 Phys. Rev. B 70064426

[2] Kaneko Y, Arima T, He J P, Kumai R and Tokura Y 2004 J. Magn. Magn. Mater. 272-276 555-6

[3] Remeika J P 1960 J. Appl. Phys. 31 S263-4 
[4] Mukherjee S, Ranjan V, Gupta R and Garg A 2011 arXiv: $1107.3623 \mathrm{v} 1$

[5] Nowlin C H and Jones R V 1963 J. Appl. Phys. 34 1262-3

[6] White D L 1960 Bull. Am. Phys. Soc. 5189

[7] Wood E 1960 Acta Crystallogr. 13682

[8] Abrahams S C, Reddy J M and Bernstein J L 1965 J. Chem. Phys. 42 3957-68

[9] Kang K Uk, Kim S B, An S Y, Cheong S W and Kim C S 2006 J. Magn. Magn. Mater. 304 e769-71

[10] Mohamed M B, Senyshyn A, Ehrenberg H and Fuess H 2010 J. Alloys Compounds 492 L20-7

[11] Kim W, We J H, Kim S J and Kim C S 2007 J. Appl. Phys. $10109 \mathrm{M} 515$

[12] Abrahams S C and Reddy J M 1964 Phys. Rev. Lett. 13688

[13] Han M J, Ozaki T and Yu J 2007 Phys. Rev. B 75060404

[14] Jones R O and Gunnarsson O 1989 Rev. Mod. Phys. 61689

[15] Kresse G and Furthmüller J 1996 Phys. Rev. B 5411169

[16] Kresse G and Joubert D 1999 Phys. Rev. B 591758

[17] Blöchl P E 1994 Phys. Rev. B 5017953

[18] Kohn W and Sham L J 1965 Phys. Rev. 140 A1133

[19] Anisimov V I, Aryasetiawan F and Lichtenstein A I 1997 J. Phys.: Condens. Matter 9767

[20] Neaton J B, Ederer C, Waghmare U V, Spaldin N A and Rabe K M 2005 Phys. Rev. B 71014113

[21] Cai M Q, Liu J C, Yang G W, Cao Y L, Tan X, Chen X Y, Wang Y G, Wang L L and Hu W Y 2007 J. Chem. Phys. 126154708

[22] Dudarev S L, Botton G A, Savrasov S Y, Humphreys C J and Sutton A P 1998 Phys. Rev. B 571505
[23] Press W H, Flannery B P, Teukolsky S A and Vetterling W T 1986 Numerical Recipes: The Art of Scientific Computing (New York: Cambridge University Press)

[24] Monkhorst H J and Pack J D 1976 Phys. Rev. B 135188

[25] King-Smith R D and Vanderbilt D 1993 Phys. Rev. B 471651

[26] Perdew J P, Ruzsinszky A, Csonka G, Vydrov O A, Scuseria G E, Constantin L A, Zhou X and Burke K 2008 Phys. Rev. Lett. 100136406

[27] Rodríguez-Carvajal J 1993 Physica B 192 55-69

[28] Yoshioka S, hayashi H, Kuwabara A, Oba F, Matsunaga K and Tanaka I 2007 J. Phys.: Condens. Matter 19346211

[29] Momma K and Izumi F 2008 J. Appl. Crystallogr. 41 653-8

[30] Baur W H 1974 Acta Crystallogr. B 301195

[31] Hoppe R, Voigt S, Glaum H, Kissel J, Muller H P and Bernet K 1989 J. Less-Common Met. 156105

[32] Ghosez P, Gonze X, Lambin P and Michenaud J P 1995 Phys. Rev. B 516765

[33] Roy A, Prasad R, Auluck S and Garg A 2010 J. Phys.: Condens. Matter 22165902

[34] Sun Z H, Dai S, Zhou Y L, Cao L Z and Chen Z H 2008 Thin Solid Films $\mathbf{5 1 6}$ 7433-6

[35] Medeiros S K, Albuquerque E L, Maia J F F, Caetano E W S, Farias G A, Freire V N, Cavada B S, Pessati M L and Pessati T L P 2005 Microelectron. J. 36 1058-61

[36] Robertson J, Xiong K and Clark S J 2006 Thin Solid Films $4961-7$

[37] Savin A, Nesper R, Wengert S and Fässler T F 1997 Angew. Chem. Int. Edn Engl. 36 1808-32

[38] Cohen R E 2008 Piezoelectricity (Springer Series in Materials Science vol 114) (Berlin: Springer) p 471 